This item was submitted to Loughborough's Research Repository by the author.

Items in Figshare are protected by copyright, with all rights reserved, unless otherwise indicated.

\title{
Quantum-classical crossover of a field mode
}

PLEASE CITE THE PUBLISHED VERSION

PUBLISHER

(C) American Physical Society

VERSION

AM (Accepted Manuscript)

LICENCE

CC BY-NC-ND 4.0

REPOSITORY RECORD

Everitt, Mark J., W.J. Munro, and T.P. Spiller. 2019. "Quantum-classical Crossover of a Field Mode”. figshare. https://hdl.handle.net/2134/4404. 
This item was submitted to Loughborough's Institutional Repository (https://dspace.lboro.ac.uk/) by the author and is made available under the following Creative Commons Licence conditions.

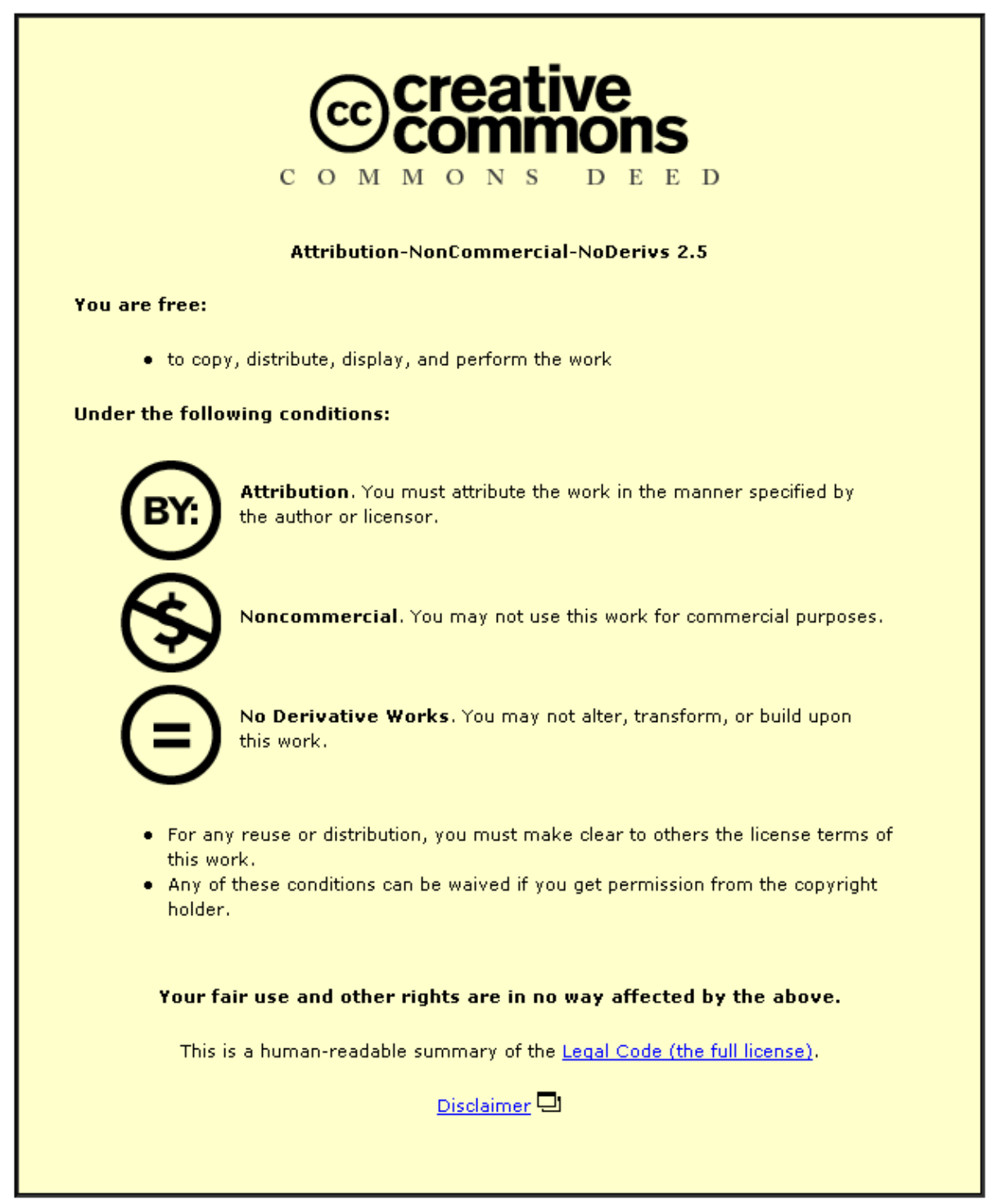

For the full text of this licence, please go to: http://creativecommons.org/licenses/by-nc-nd/2.5/ 


\title{
The quantum-classical crossover of a field mode
}

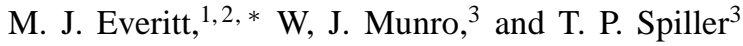 \\ ${ }^{1}$ Centre for Theoretical Physics, The British University in Egypt, El Sherouk City, Postal No. 11837, P.O. Box 43, Egypt. \\ ${ }^{2}$ Department of Physics, Loughborough University, Loughborough, Leics LE11 3TU, United Kingdom \\ ${ }^{3}$ Hewlett-Packard Laboratories, Filton Road, Stoke Gifford, Bristol BS34 8QZ, United Kingdom
}

(Dated: March 24, 2009)

\begin{abstract}
We explore the quantum-classical crossover in the behaviour of a quantum field mode. The quantum behaviour of a two-state system-a qubit - coupled to the field is used as a probe. Collapse and revival of the qubit inversion form the signature for quantum behaviour of the field and continuous Rabi oscillations form the signature for classical behaviour of the field. We demonstrate both limits in a single model for the full coupled system, for field states with the same average field strength, and so for qubits with the same Rabi frequency.

PACS numbers: 03.65.-w,03.65.Ta,03.65.Yz,03.67.-a,42.50.-p
\end{abstract}

There has long been interest in how fundamental quantum things give rise to the classical behaviour much more familiar to us in our everyday lives. Since the time of "Ehrenfest's theorem" [1] it has been known that expectation values follow their corresponding classical mechanical equations. However, this isn't the whole story, and so most of us seek rather more than just this, since spreading quantum waves hardly look like classical lumps, or particles.

Quantum measurement [2] has played an extremely important role in this area. Clearly it isn't possible to observe any form-quantum, or tending to classical—of behaviour of a quantum system without measurement. It therefore makes sense to consider the role of measurement apparatus, or the degrees of freedom that are used to observe the behaviour of the system of interest. An early example of this is the work of Neville Mott on $\alpha$-ray tracks [3].

The role of decoherence in general is also very important in this area [4, 5]. Loosely speaking, since measurement interactions - discrete and projective, or continuous - can be thought of as localising quantum states (so spreading waves begin to look more like particles following trajectories), other forms of decoherence that provide similar localisation effects contribute to quantum systems behaving more classically. Indeed, within this picture, in which quantum measurement is regarded as just one example of decoherence applied to a system, it may be that other forms of decoherence, such as dissipative coupling to an environment, are dominant. So these sorts of decoherence alone may effect the classical limit of a quantum system's behaviour, whether or not anyone (with or without a $\mathrm{PhD}$ [6]) is looking.

One explicit example of this is the emergence of chaos in dissipative quantum systems [7, 8, 9, 10, 11, 12, 13, 14]. Non-linear dissipative and driven classical systems can exhibit chaos, demonstrated, for example, through a periodic phase space plot showing a strange attractor, as opposed to isolated points corresponding to regular motion. Various examples have been given [7, $8,9,10,11,12,13,14]$ of the emergence of such chaos - a characteristic of classical dynamical motion-in the corresponding dissipative and driven quantum

\footnotetext{
*Electronic address: m.j.everitt@physics.org
}

systems. These examples generally involve scaling the parameters governing the quantum dynamics so that the phase space motion becomes large on the scale of $\hbar$ and the quantum state starts to look like a localised lump following a classical trajectory. Similar behaviour can emerge in Hamiltonian chaotic systems, suitably measured [15].

In the work presented here, we focus on the example of an oscillator, or field mode. We'll show how both characteristic classical and quantum behaviour can emerge from the single framework of a dissipative quantum model, as appropriate parameters are varied. In order to achieve this, we couple a twolevel quantum system/atom, or qubit, to the field mode. In effect, the qubit is used to monitor the regime of behaviour of the field. This simple model is applicable to a broad range of physical systems - atomic or fabricated qubits coupled to an optical cavity; atomic or charge qubits coupled to a microwave cavity; charge or magnetic qubits coupled to a nanomechanical resonator.

The classical limit of the field behaviour is demonstrated through continuous Rabi oscillations of the qubit [16, 17]. A qubit, driven by an appropriate oscillatory classical field, oscillates between its two energy eigenstates. If these are taken to be the eigenstates of the $\sigma_{z}$ Pauli matrix, separated by energy $\hbar \omega_{0}$, an appropriate Hamiltonian for the qubit coupled to an external classical field of frequency $\omega$ is

$$
H_{c l}=\frac{\hbar \omega_{0}}{2} \sigma_{z}+\hbar \nu \cos \omega t \sigma_{x}
$$

The detuning is defined as $\Delta=\omega_{0}-\omega$ and the Rabi oscillation frequency as $\Omega_{R}=\sqrt{\Delta^{2}+\nu^{2}}$, so for the case of the field on resonance with the qubit (zero detuning, $\Delta=0$ ) the Rabi frequency is simply $\Omega_{R}=\nu$. It is set by the amplitude of the field, not its frequency. In this resonant case, a qubit initially in state $\left|\uparrow_{z}\right\rangle$ oscillates fully to $\left|\downarrow_{z}\right\rangle$ at frequency $\Omega_{R}$. In the language of atomic and optical physics, the atomic inversion-in qubit language $\left\langle\sigma_{z}\right\rangle$-satisfies $\left\langle\sigma_{z}\right\rangle=\cos \Omega_{R} t$. In the absence of any decoherence acting on the qubit, these Rabi oscillations persist and are a well known characteristic of a qubit resonantly coupled to an external classical field. An example is shown in figure 1.

The quantum limit of the field behaviour is demonstrated through collapse [18, 19, 20] and revival [17, 21, 22, 23] of the qubit Rabi oscillations. If, instead of treating the field as 
a classical source in the Hamiltonian of the qubit, the field is treated as a quantum oscillator mode Jaynes-Cummings Hamiltonian [17, 24] provides the appropriate description

$$
H_{q}=\frac{\hbar \omega_{0}}{2} \sigma_{z}+\hbar \omega\left(a^{\dagger} a+\frac{1}{2}\right)+\hbar \lambda\left(\sigma_{+} a+\sigma_{-} a^{\dagger}\right) .
$$

Here $a^{\dagger}(a)$ is the creation (annihilation) operator for the field, with $\left[a, a^{\dagger}\right]=1, \sigma_{ \pm}=\frac{1}{2}\left(\sigma_{x} \pm i \sigma_{y}\right)$ are the qubit operators that effect transitions between the energy $\left(\sigma_{z}\right)$ eigenstates, and $\hbar \lambda$ is the coupling energy between the qubit and the field. In order to derive the Rabi oscillations in a classical field analytically, it is usual to chuck away the rapidly rotating terms. This (very good) rotating wave approximation is also made in the derivation of equation (2) from the underlying dipole interaction between the qubit and field, so both quantum and classical models are on the same footing. In the quantum case, a qubit initially in state $\left|\uparrow_{z}\right\rangle$ is coupled to a coherent state of the field

$$
|\alpha\rangle=e^{-|\alpha|^{2} / 2} \sum_{n=0}^{\infty} \frac{\alpha^{n}}{\sqrt{n !}}|n\rangle,
$$

where the basis $\{|n\rangle\}$ comprises the Fock, or number, states of the field. We note that $|\alpha\rangle$ is an eigenstate of the annihilation operator labelled by its eigenvalue $\alpha$. In this scenario, although the qubit initially exhibits Rabi oscillations analogous to those in the classical case, these apparently "decay", and then subsequently revive [17, 21, 22, 23]. An example is shown in figure 1. Such collapse and revival of Rabi oscillations of a qubit is widely recognised as a characteristic of a qubit coupled to a quantum field mode. It is understood both theoretically [25, 26, 27] and experimentally [28, 29] that the

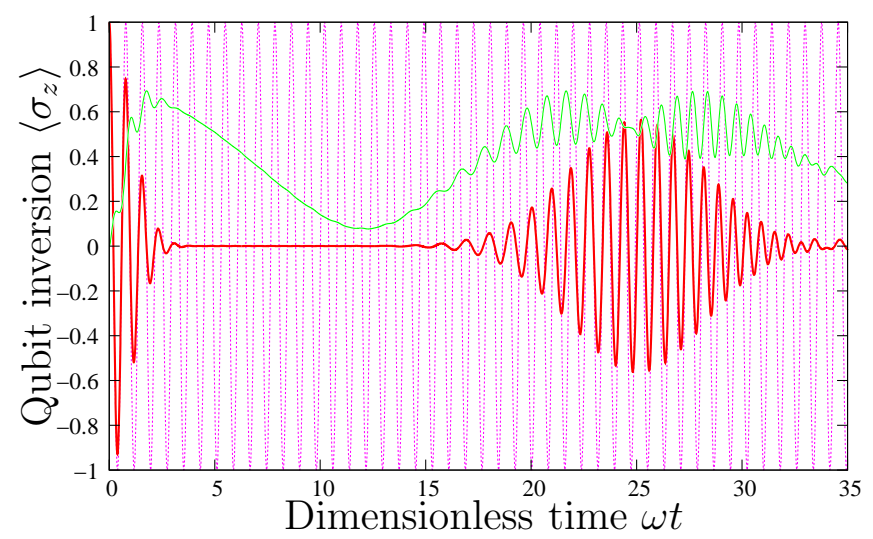

FIG. 1: (Color online) Qubit inversion, $\left\langle\sigma_{z}\right\rangle$, as a function of dimensionless time $\omega t$ for the resonant cases of Rabi oscillations (dotted; light grey/magenta) in a classical field and collapse and revival (dark grey/red heavy line) in a quantum field. For the classical field case $\nu / \omega=8$ is used, corresponding to the same dominant Rabi frequency (see [17]) as in the quantum field case, which uses a coherent state (3) with $\alpha=\sqrt{15}$ and $\lambda / \omega=1$. Also shown (in green/light grey solid line) is the qubit entropy (in Nats), which indicates the degree of entanglement with the field. apparent decay of qubit coherence is due to entanglement with the field mode, generated by the coherent evolution of the coupled quantum systems. This is illustrated in figure 1 through a plot of the qubit entropy, $S(t)=-\operatorname{Tr}(\rho(\mathrm{t}) \ln \rho(\mathrm{t}))$ where $\rho(t)$ is the reduced density matrix of the qubit resulting from a trace over the field (or vice versa, given the initial system state is pure). Clearly there is a sharp rise in entropy sympathetic with the initial collapse. The qubit then disentangles from the field at the "attractor time" [25, 26], half way to the revival. The revival arises through oscillatory re-entanglement of the qubit and field, as seen through the subsequent entropy oscillations that coincide with the revival. Obviously there is no entanglement between the qubit and the field in the classical limit, because the field is classical, so the qubit entropy is zero for all times.

It is well known that there are three timescales in the collapse and revival situation [17]. For fields with large $\langle n\rangle=$ $\bar{n}$, the Rabi time (or period) is given by $t_{R}=2 \pi \Omega_{R}^{-1}=$ $\pi /(\lambda \sqrt{\bar{n}})$, the collapse time that sets the Gaussian decay envelope of the oscillations by $t_{c}=\sqrt{2} / \lambda$ and the (first) revival time that determines when the oscillations reappear, such as in the example of figure 1, by $t_{r}=2 \pi \sqrt{\bar{n}} / \lambda$. For the coherent state (3) the average photon, or excitation, number is $\bar{n}=|\alpha|^{2}$. Note that the different dependencies of the times on $\sqrt{\bar{n}}$ (which corresponds to the-e.g. electric-field strength of the coherent field state) allow a sort of "classical limit" to be taken. As $\bar{n}$ is increased, there are more Rabi periods packed in before the collapse-so this appears more like persistent Rabi oscillations - and the revival is pushed out further in time. However, the collapse (and revival) still occur eventually, and in any case the reason there are more Rabi oscillations before the collapse is due to the inverse scaling of $t_{R}$ with $\sqrt{n}$, so the actual Rabi period is shortened as $\bar{n}$ is increased. The classical limit we consider in this work is quite different. We shall consider a fixed $\bar{n}$, so the Rabi period of the qubit does not change in our various examples. We'll show how the transition from quantum (collapse and revival) to classical (continuous Rabi oscillations) can be effected by introducing decoherence to the quantum field. Our work complements the dissipative, small- $n$, short-time study of Kim et al. [30], who show that such fields are sufficiently classical to provide Ramsey pulses to Rydberg atoms.

Next we introduce and discuss the decoherence applied to the quantum field model, used to push it into a regime of classical behaviour. We use the very simple model -it's all we need to make the demonstration —of a Lindblad [31] or Bloch type master equation. Here, the evolution of the density operator of the quantum system of interest, $\rho(t)$, depends only on $\rho(t)$ and not its history, so there are no memory effects (i.e. the evolution is Markovian),

$\dot{\rho}=-\frac{i}{\hbar}\left[H_{q}, \rho\right]+\sum_{m}\left(L_{m} \rho L_{m}^{\dagger}-\frac{1}{2} L_{m}^{\dagger} L_{m} \rho-\frac{1}{2} \rho L_{m}^{\dagger} L_{m}\right)$

The evolution generated by equation (4) is irreversible and non-unitary. The first term is the conventional Schrödinger evolution, but the terms due to the operators $\left\{L_{m}\right\}$ introduce the irreversibility. These can be thought of as the (approxi- 
mate) by-product of coupling the quantum system of interest to an environment- $a$ bath of other quantum degrees of freedom. This environment introduces decoherence in the evolution of the (reduced) density operator of the quantum system of interest.

In our case, we introduce damping to the quantum field, but no direct decoherence to the qubit. So we use just a single operator, $L=\sqrt{\gamma} a$, where $\gamma$ is the decay constant. Implicitly we work at zero temperature, although the work could easily be extended to finite temperature (introducing thermal noise on top of quantum noise in the environment) by use of a second operator proportional to $a^{\dagger}$ and suitably chosen (temperaturedependent) coefficients of both operators. This damping form of decoherence is quite generic. Examples of model environments that give such damping are a bath of two-level atoms (qubits), or a bath of harmonic oscillator modes. For a field mode in a cavity, these environments respectively represent a lossy atomic medium in the cavity, or external modes to which the field can leak. For explicit derivations of equation (4), giving damping for a field mode coupled to such baths, see reference [32].

In order to explore the full spectrum of quantum through to classical behaviour of the field, at a fixed Rabi frequency, we need to use a field strength which is significantly larger than the simple examples of figure 1 The field strength has to be large compared to the quantum uncertainty in the field, so it can be pushed into a good classical limit, which requires $\bar{n} \gg 1$. However the field strength cannot be so large that the collapse and revival computations in the quantum limit become intractable. We have therefore used $\bar{n}=50$ for the examples presented here. A further constraint is the use of weak coupling. In the usual discussions of collapse and revival [17], the coupling $\lambda$ in equation (2) simply scales the time axis, as all three times $t_{R}, t_{c}$ and $t_{r}$ scale as $\lambda^{-1}$. However, when decoherence is applied to the field mode, in order to get clean signatures from our qubit probe, it is desirable to couple it weakly to the field. Also, thinking perturbatively, the back-reaction of the qubit on the field has to be weak to enable the field to approach the limit of a fixed classical source in our driven dynamical model. We therefore use weak coupling of $\lambda / \omega=0.0005$.

The large $\bar{n}$ and weak coupling regime in which we work equates to some pretty heavyweight computing. Rather than solving the master equation (4) directly, which would be a substantial matrix evolution for a very long time, we therefore choose to solve an unravelling state evolution equation for the system, equivalent to (4). This only requires solution of state vector, rather than density matrix, evolution. The unravelling we use is quantum state diffusion (QSD) [33, 34]. In an unravelling description, a quantum state $|\psi(t)\rangle$ is used, such that the density operator is recovered from $\rho(t)=M(|\psi(t)\rangle\langle\psi(t)|)$ where $M$ denotes the mean over an ensemble. For QSD, the state evolves according to

$$
\begin{aligned}
& |d \psi\rangle=-\frac{i}{\hbar} H_{s}|\psi\rangle d t+\sum_{m}\left(L_{m}-\left\langle L_{m}\right\rangle_{\psi}\right)|\psi\rangle d \xi_{m}+ \\
& \sum_{m}\left(\left\langle L_{m}^{\dagger}\right\rangle_{\psi} L_{m}-\frac{1}{2} L_{m}^{\dagger} L_{m}-\frac{1}{2}\left\langle L_{m}^{\dagger}\right\rangle_{\psi}\left\langle L_{m}\right\rangle_{\psi}\right)|\psi\rangle d t
\end{aligned}
$$

where the operators are defined as in (4) and the $d \xi_{m}$ are independent complex differential random variables satisfying $M\left(d \xi_{m}\right)=M\left(d \xi_{m} d \xi_{n}\right)=0$ and $M\left(d \xi_{m}^{*} d \xi_{n}\right)=\delta_{m n}$. The evolution of (5) is equivalent to that of (4) when the ensemble mean over the noise is taken. However, it is also the case that in certain situations where the effects of the noise are small, such as the quantum limit where the decoherence is very weak, or a classical limit where the motion is large compared to the noise, a single run of a state unravelling essentially provides a reasonable approximation to the full ensemble average, at least for non-chaotic systems. We utilise this for our work here, as these limits are what interest us and, for the parameters we employ, full ensemble averaging out to and past the revival time is computationally infeasible.

In order that the quantum field always follows an evolution that gives rise to our chosen Rabi frequency for the probe qubit, as we add damping to the field to make it more classical, we also need to add an external drive term to maintain it, so it continues to oscillate with the chosen value of $\bar{n}$. For damping with a decay constant $\gamma$, this requires a drive term applied to the field of $H_{d}=-\hbar \gamma \alpha \cos \omega t\left(a+a^{\dagger}\right)$. As the field is always started in the coherent state $|\alpha\rangle$, there are no transients as it settles down to its damped, driven trajectory. In the limit that there is no decoherence acting, there is also no drive, and we shall recover the quantum limit of collapse and revival.

Before presenting our results, we note two further points. (i) Introducing damping through the master equation does not produce the expected shift in oscillator frequency, which for our definition of $\gamma$ is $\omega \rightarrow \sqrt{\omega^{2}-\frac{\gamma^{2}}{4}}$. Therefore, in order to ensure the correct behaviour of the field mode in the presence of damping we also need to add a further term to the Hamiltonian (2): $\frac{\hbar \gamma i}{4}\left(a^{\dagger^{2}}-a^{2}\right)$. We note that in the literature this term is usually expressed in terms of position $q$ and momentum $p$ operators in the form $\frac{\gamma}{4}(q p+p q)$, (see for example [8, 34]).

(ii) Detailed studies have been made (see for example [35, 36]) of the Jaynes-Cummings system with dissipation applied to the field, resulting in photon loss. For our investigation of the classical limit, we require no net photon loss (to maintain the chosen Rabi frequency). Therefore it is crucial that we also include a suitable drive term, to compensate and prevent damping of the field. This driving is, of course, what is done in experiments in order to apply a constant amplitude classical field to a quantum system using a lossy resonator.

So now to our results. In figure 2 we show the qubit inversion $\left\langle\sigma_{z}\right\rangle$ for various values of damping (and thus also drive) applied to the quantum field, each computed using a single run of QSD. For the field part of the system to behave classically its coherence time should be short compared to the Rabi time, so $\gamma \gg \lambda \sqrt{\bar{n}} / \pi\left(\sim 10^{-3}\right.$ for our parameters), as in the top left plot. For the field to approach the quantum limit, its coherence time should exceed the revival time, so $\gamma \ll \lambda /(2 \pi \sqrt{\bar{n}})\left(\sim 10^{-5}\right.$ for our parameters), as in the bottom right plot. The pure undamped collapse and revival behaviour is accurately reproduced in this limit. In both the quantum and classical limits, just a single run of QSD gives a 

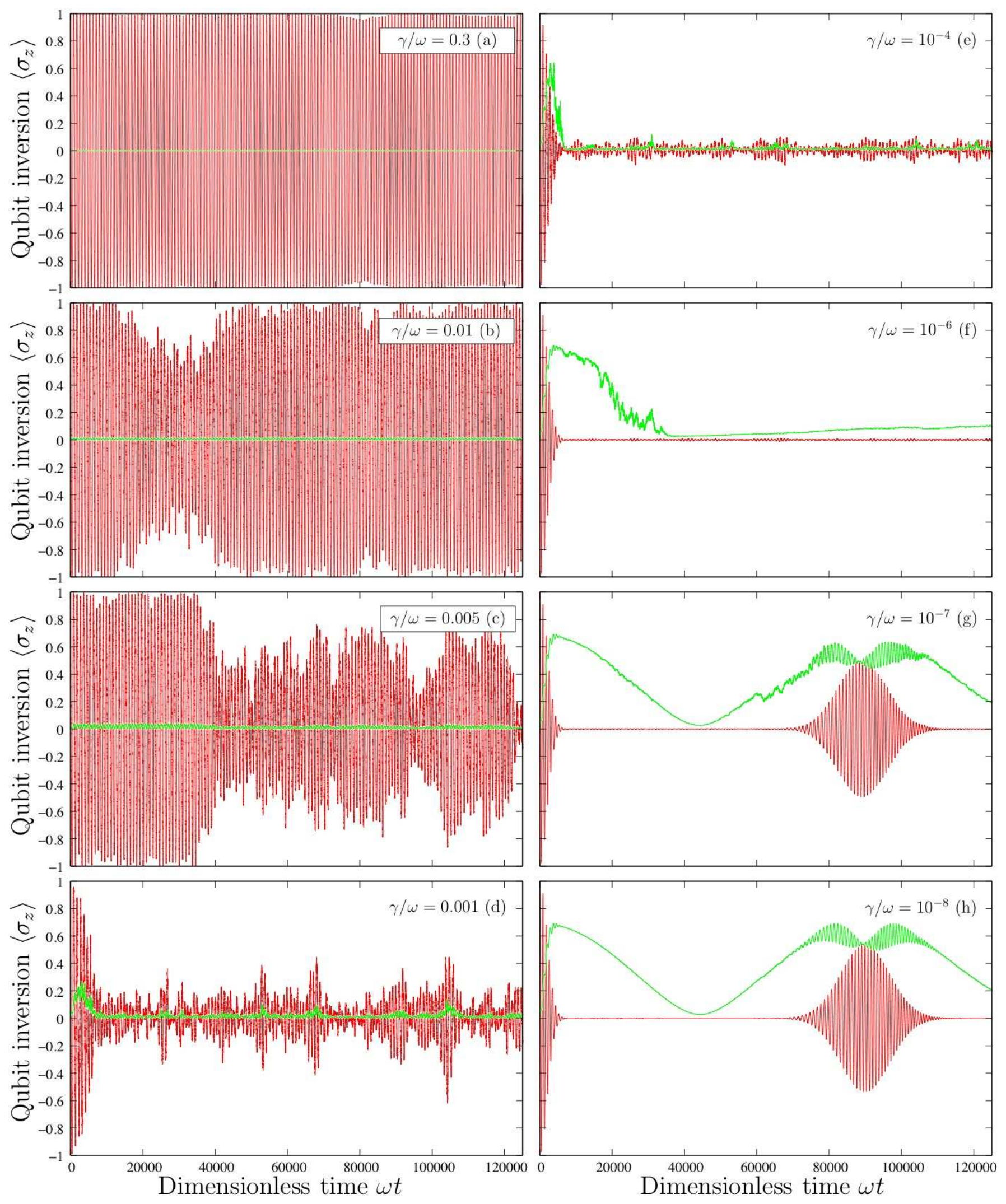

FIG. 2: (Color online) Results (dark grey/red) showing qubit inversion, $\left\langle\sigma_{z}\right\rangle$, for different values of dissipation (and therefore drive) applied to the field. These illustrate collapse and revival, suppression of collapse and an approach to complete Rabi oscillations as dissipation and drive are increased. For each individual QSD run shown, the qubit entropy in Nats (light grey/green) is superimposed. 
very reasonable representation of the qubit inversion, because the effect of the quantum noise is small. The intermediate plots are in the quantum-classical crossover region. Here the quantum noise is significant - a full ensemble average (which is beyond our numerical capability) would be needed to give the actual qubit inversion. Nevertheless, even in these example individual QSD runs there is evidence of the qubit Rabi oscillations extending beyond the basic collapse of the quantum limit, as the decoherence of the field is increased.

In our approach, there are two ways in which the qubit state could become mixed. Firstly, it could entangle with the field [25, 26, 27, 28, 29], as happens in the pure quantum limit to generate the collapse. Such entanglement can be inferred from the qubit entropy in a single run of QSD (for which the full qubit-field state is pure). Secondly, the qubit could remain pure in an individual QSD run, but, when averaged over an ensemble, show mixture. For the classical limit of the top left plot of figure 2, we have calculated that both of these effects are small for times in excess of the collapse time. The qubitfield entanglement remains very close to zero for all times in a single QSD run, as shown in the entropy plot presented. Independent QSD runs have been made and these show that the qubit mixture is still very small at the collapse time. Therefore the persistence of good Rabi oscillations well beyond the collapse time and all the way out to the revival time, as illustrated in the top left plot of figure 2, provides a clear signature of the classical limit of the field. In this limit, the quantum field state is a localized lump in phase space (like a coherent state), following the expected classical trajectory and suffering negligible back-reaction from the qubit. However, the field coherence time is so short as to prevent entanglement with the qubit developing, unlike in the quantum limit [25, 26, 27, 28, 29]. The resultant qubit Rabi oscillations are thus like those due to a classical field, and not like those [17] that arise from entanglement with a single Fock state (which is a delocalized ring in phase space).

Further insight into the classical limit can be obtained from the phase space behaviour of the field. It is well known that in the pure quantum limit $[25,26,27,28,29]$ the qubit-field entanglement correlates distinct and localised (coherent-like) states of the field with different qubit amplitudes. Thus, when there is no entanglement at the "attractor time", the interaction of the atom with the field mode generates a macroscopically distinct superposition of states in the oscillator-a Schrödinger cat state. As one would expect, and in order to render the field behaviour classical, the introduction of decoherence suppresses this phenomenon. We illustrate this in [37] by providing two animations of the dynamics of the Wigner function and atomic inversion for the parameters of Fig. 1, one undamped and one with dissipation of $\gamma / \omega=0.01$.

In the quantum limit, the bottom right of figure 2 the quantum noise in the single run of QSD is so small that the entropy of the qubit in this single run gives a very reasonable approximation to the entropy of the ensemble, which matches well with the qubit entropy in the exactly zero dissipation limit. Figure 3 gives focus to the parameter range where the revival begins to emerge. For all the individual QSD runs that are neither well into the classical or well into the quantum limit, we

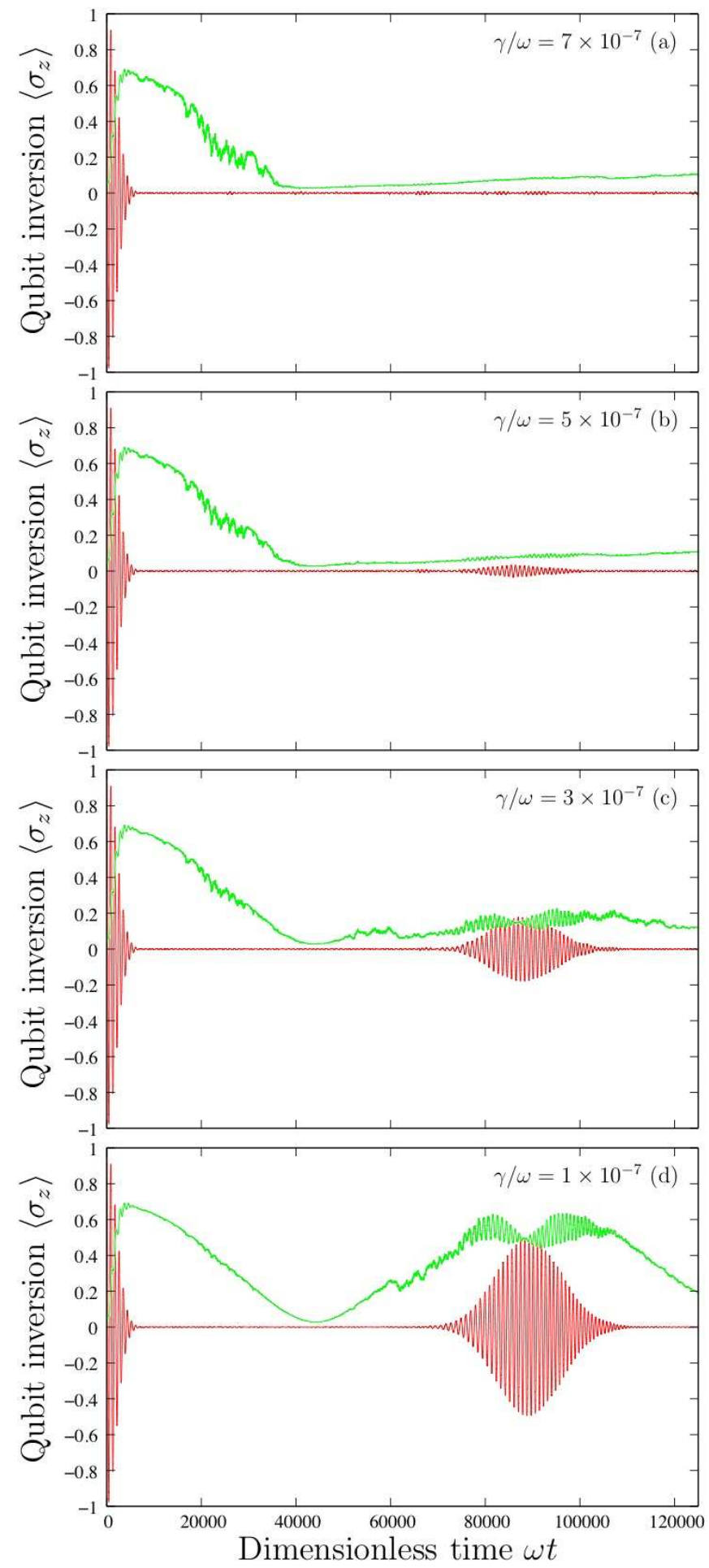

FIG. 3: (Color online) Results (dark grey/red) showing qubit inversion, $\left\langle\sigma_{z}\right\rangle$, for different values of dissipation (and therefore drive) applied to the field. These plots focus on the parameter range where the revival of Rabi oscillations begins to emerge. For each individual QSD run shown, the qubit entropy in Nats (light grey/green) is superimposed. 
still plot the qubit entropy for that run. It should be understood that these do not approximate well the ensemble entropy in this crossover parameter regime. Nevertheless, as the damping and drive applied to the field are reduced, these plots do illustrate the emergence of an entropy peak sympathetic with the collapse, an entropy dip at the "attractor time" [25, 26] (half the revival time), and the emergence of entropy oscillations sympathetic with the revival.

In conclusion, we have shown how to take a quantum field through the quantum-classical crossover, as seen through the behaviour of a coupled qubit, used as a probe. Crucially, this crossover is not made through increasing the field strength; rather, it is made at a fixed field strength, through adding dissipation to the field. So the qubit Rabi frequency is fixed through the whole range of behaviour shown. Thus a quantum field with around fifty photons in it can be made to act rather classically, or still show its characteristic quantum properties, dependent upon the environment coupled to it. Perhaps amusingly, the apparent coherence of the probe qubit is increased by adding dissipation to the field to which it is coupled. This is demonstrated through increased persistence of the qubit Rabi oscillations, or suppression of the collapse. Our work provides an explicit example of how it is possible to decohere and make classical part of a coupled system, butcrucially - without that decoherence leaking through to decohere the other, still quantum, part of the system. The classical part of the system loses its ability to entangle with the still quantum part, but in this case does not destroy the coherence of the probe qubit. As stressed in the introduction, the model used here is relevant for a broad range of physical systems. To fully explore the quantum-classical crossover in experiments, the "intrinsic" coherence time of the probe qubit (limited by all other environment effects, not its coupling to the field) must exceed the revival time. This is likely to be very challenging in current experiments. However, evidence for the "suppression of collapse" only requires the intrinsic qubit coherence time to exceed the collapse time. This is much less demanding, and provides an initial goal for current qubit-field experiments of various kinds.

\section{Acknowledgments}

We thank Catherine Jarvis for helpful discussions.
[1] P. Ehrenfest, Z. Physik 45, 455 (1927).

[2] J. A. Wheeler and W. H. Zurek (eds.), Quantum Theory and Measurement, (Princeton University Press, 1983).

[3] N. F. Mott, Proc. Roy. Soc. Lond. A 126, 79 (1929).

[4] E. Joos and H. D Zee, Z. Phys. B 59, 223 (1985).

[5] W. H. Zurek, Physics Today 44(10), 36 (1991); see also arXiv:quant-ph/0306072

[6] J. S. Bell, Physics World 3 (8), 33 (August 1990).

[7] T. P. Spiller and J. F. Ralph, Phys. Lett. A 194, 235 (1994).

[8] T. A. Brun, I. C. Percival and R. Schack, J. Phys. A 29, 2077 (1996).

[9] S. Habib, K. Shizume and W. Zurek, Phys. Rev. Lett. 80, 4361 (1998).

[10] M. J. Everitt, T. D. Clark, P. B. Stiffell, J. F. Ralph, A. R. Bulsara and C. J. Harland, Phys. Rev. E 72, 066209 (2005).

[11] S. Dyrting and G.J. Milburn, Quantum and Semiclassical Optics 8, 541-555 (1996)

[12] T. Bhattacharya, S. Habib and K. Jacobs, Phys. Rev. Lett. 85, $4852-4855$ (2000)

[13] S. Habib, K. Jacobs and K. Shizume, Phys. Rev. Lett. 96, 010403 (2006)

[14] A. Kapulkin and Arjendu K. Pattanayak, Phys. Rev. Lett. 101, 074101 (2008)

[15] M. J. Everitt, Phys. Rev. E 75, 036217 (2007).

[16] I. I. Rabi, Phys. Rev. 51, 652 (1937).

[17] C. C. Gerry and P. L. Knight, Introductory Quantum Optics, chapter 4 (Cambridge University Press, 2005).

[18] F. W. Cummings, Phys. Rev. 149, A 1051 (1965).

[19] S. Stenholm, Phys. Rep. 6, 1 (1973).

[20] P. Meystre, E. Geneux, A Quattropani and A. Faist, Nuovo Cim. B 25, 521 (1975); T von Foerster, J. Phys. A 8, 95 (1975).

[21] J. H. Eberly, N. B. Narozhny and J. J. Sanchez-Mondragon, Phys. Rev. Lett. 44, 1323 (1980).
[22] N. B. Narozhny, J. J. Sanchez-Mondragon and J. H. Eberly, Phys. Rev. A 23, 236 (1981).

[23] P. L. Knight and P. M. Radmore, Phys. Rev. A 26, 676 (1982).

[24] E. T. Jaynes and F. W. Cummings, Proc. IEEE 51, 89 (1963).

[25] J. Gea-Banacloche, Phys. Rev. Lett. 65, 3385 (1990).

[26] J. Gea-Banacloche, Phys. Rev. A 44, 5913 (1991).

[27] V. Bužek and P. L. Knight, Prog. Opt. 34, 1 (1995).

[28] A. Auffeves, P. Maioli, T. Meunier, S. Gleyzes, G. Nogues, M. Brune, J. M. Raimond and S. Haroche, Phys. Rev. Lett. 91, 230405 (2003).

[29] T. Meunier, S. Gleyzes, P. Maioli, A. Auffeves, G. Nogues, M. Brune, J. M. Raimond and S. Haroche, Phys. Rev. Lett. 94, 010401 (2005).

[30] J. I. Kim, K. M. Fonseca Romero, A. M. Horiguti, L. Davidovich, M. C. Nemes and A. F. R. de Toledo Piza, Phys. Rev. Lett. 82, 4737 (1999).

[31] G. Lindblad, Commun. Math. Phys. 48, 119 (1976).

[32] M. Sargent III, M. O. Scully and W. E. Lamb Jr., it Laser Physics, chapters 16, 17 (Addison-Wesley Publishing Company, 1974; fifth printing 1987).

[33] N. Gisin and I. C. Percival, J. Phys. A 25, 5677 (1992).

[34] I. C. Percival, Quantum State Diffusion, (Cambridge University Press, 1998).

[35] B. Daeubler, H. Risken and L. Schoendorff, Phys. Rev. A 46, 1654 (1992).

[36] J. Gea-Banacloche, Phys. Rev. A 47, 2221 (1993).

[37] See EPAPS Document No. [number will be inserted by publisher] for animations of the Wigner function and atomic inversion for the papameters of Fig. 1, one undamped and one with dissipation of $\gamma / \omega=0.01$. For more information on EPAPS, see http://www.aip.org/pubservs/epaps.html. 